Document downloaded from:

http://hdl.handle.net/10251/103248

This paper must be cited as:

Castillo-Frasquet, A.; García Gil, PJ.; Sanz Diaz, R.; Albertos Pérez, P. (2017). Enhanced extended state observer-based control for systems with mismatched uncertainties and disturbances. ISA Transactions. 73:1-10. doi:10.1016/j.isatra.2017.12.005

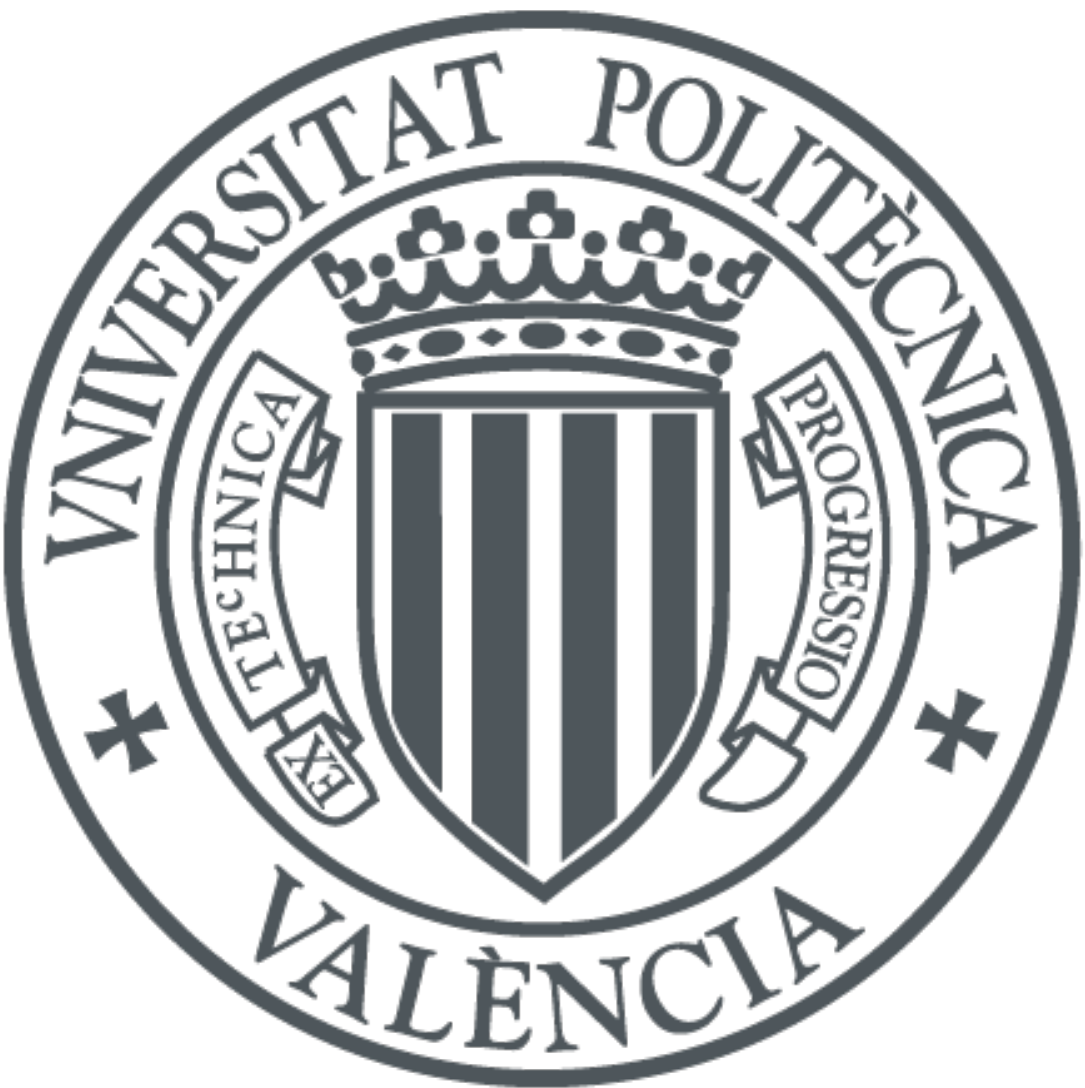

The final publication is available at

https://doi.org/10.1016/j.isatra.2017.12.005

Copyright Elsevier

Additional Information 


\title{
Enhanced Extended State Observer-Based Control for Systems with Mismatched Uncertainties and Disturbances
}

\author{
A. Castilloa, P. García ${ }^{a}$, R. Sanz ${ }^{\text {a }}$, P. Albertos ${ }^{\text {a }}$ \\ ${ }^{a}$ Instituto de Automática e Informática Industrial, Universitat Politècnica de València, 46020 Valencia, Spain.
}

\begin{abstract}
This paper presents an enhanced Extended State Observer (ESO)-based control strategy to deal with the disturbance attenuation problem for a class of non integral-chain systems subject to non-linear mismatched uncertainties and external disturbances. The proposed control strategy does not assume the integral-chain form and it is formed by a state-feedback plus a dynamic disturbance compensation term, which is designed to reject the disturbance effect in the system output. From a theoretical point of view, the proposed strategy is reduced to the conventional ESO when the integral chain form and the matched condition hold. In this sense, this paper is presented as an extension of the ESO principles to cover a wider class of systems. The theoretical results show that the internal zero-dynamics plays an important role in ESObased control design. Also, the closed-loop stability is analyzed and some numerical simulations show the effectiveness of the proposal in comparison with previous ESO-based techniques.
\end{abstract}

Keywords: Extended State Observer (ESO), Generalized Extended State Observer (GESO), Active Disturbance Rejection Control (ADRC), Mismatched Uncertainty, Disturbance Rejection, Nonintegral-chain system.

\section{Introduction}

Disturbance rejection is a fundamental topic in control theory [1]. Many control systems are normally affected by unmeasurable external disturbances and/or unmodeled internal nonlinearities which may degrade the closed-loop control performance [2]. Due to the increasing interest in high precision control, the use of disturbance estimation techniques, which are capable of attenuating those uncertainties, are often useful in the controller design. The final objective is that the control operation should not be influenced by those internal or external uncertainties [3].

Many Disturbance Observer Based Control (DOBC) methods, such as the Disturbance Observer (DOB) $[4,5]$, the Equivalent Input Disturbance (EID) [6, 7], the Uncertainty and Disturbance Estimator (UDE) $[8,9,10]$ or the Extended State Observer (ESO) [11, 12], among others, have been developed with this main purpose. Their principal ideas are: i). to obtain a disturbance estimation through the plant input-output measurements and ii). to select a control action which, by means of the disturbance estimation, attenuates its effect. A detailed DOBC review can be found in the recent survey paper [1].

Although many results in DOBC have been developed for systems satisfying the so-called matched condition, there are fewer results concerning systems with mismatched uncertainties as pointed out in $[3,13,14]$. In fact,

Email addresses: alcasfra@gmail.com (A. Castillo), pggil@isa.upv.es (P. García), risanzdi@gmail.com (R. Sanz), pedro@aii.upv.es (P. Albertos) it is mentioned in [13] that "the disturbance-based feedforward control for systems with mismatched disturbances is a longstanding unresolved problem". Indeed, it is found that in many practical systems such as magnetic levitation [15], flight control systems [16] or permanent magnet synchronous motor systems [17], the disturbance does not affect the system in the same channel than the control action. This motivates the development of DOBC techniques capable of dealing with mismatched uncertainties.

The aforementioned DOBC techniques employ some kind of plant information for disturbance observation and control design. The ESO is the one that uses the less information as only the system relative degree should be known [18]. For that reason, the ESO has become very popular in recent years. It is the core of the Active Disturbance Rejection Control (ADRC) $[18,19]$ and many studies providing theoretical analyses $[20,21,22,23]$, or practical applications [24, 25, 26, 27], have been proposed. As the knowledge of the relative degree is its unique requirement, it is natural to doubt about what kind of plant information needs to be really considered for control design [13, 28]. However, the original ESO assumes that the plant is expressed in the Conventional Integral Chain Form (CICF) satisfying the matched condition [3, 12]. Therefore, its applicability is restricted to systems which, directly or by means of a change of variable, can be expressed in the $\mathrm{CICF}$. In this sense, the advantage of requiring the less plant information for control design is contrasted with the limitation of expressing the system in the CICF for analysis purposes. Performing such transformation is not al- 
ways easy as it is mentioned in $[18,19,29]$, specially if the system has zero-dynamics. Therefore, motivated by the successful results of the ESO, it was recently pointed out in [13] that it is imperative to develop ESO-based control techniques for systems without assuming the CICF.

One of the major results in this area is presented in [13], where a novel Generalized Extended State Control (GESO) is proposed in order to extend the ESO principles to a class of systems with mismatched uncertainties that are not expressed in the CICF. Concretely, in [13], a disturbance compensation gain is designed to reject the disturbance from the system output in steady-state, while the disturbance, $f$, is assumed to satisfy $\lim _{t \rightarrow \infty} \dot{f}=0$. Other works in which the CICF is not assumed have been recently developed. In [28] an ADRC is designed for the same system considered in [13] but assuming the matched condition. In that work, it is shown that the knowledge of the inner plant dynamics led to better control performance. In [30] it is shown how the system considered in [13] can be reduced to the CICF if the plant does not have zeros. In [31], the GESO is applied to control a cartpendulum system.

This paper aims to apply the ESO principles to control a class of non integral-chain systems subject to non-linear mismatched uncertainties. The proposed control strategy does not assume the integral-chain form and it is formed by a state-feedback plus a dynamic disturbance compensation term. This strategy is able to attenuate an uncertainty whose $k_{d}+1$ time-derivative is bounded (being $k_{d}$ a positive integer unequivocally determined by the internal system structure); and it is able to reject an uncertainty satisfying $\lim _{t \rightarrow \infty} f^{\left(k_{d}+1\right)}(t)=0$. Some simulation results show that the disturbance attenuation is enhanced with this strategy in comparison with the previous ones. Also, from a theoretic point of view, this scheme can be interpreted as an extension of the GESO-based control presented in [13] in which the requirement of steady-state is avoided; or as an extension of the conventional ESO in which the requirements of the CICF and the matched condition are eliminated. This paper also shows that the knowledge of the internal zero dynamics plays an important role in ESO-based control design.

The rest of the paper is structured as follows. In Section 2 the system under consideration is presented and the GESO-based control law is recalled. Section 3 contains the main results. First, the proposed ESO-based control law is defined in general terms. Then, in Section 3.1, an analysis in order to obtain the explicit expression of the required control action so that the effect of the mismatched uncertainty is removed from the system output is performed. The closed-loop stability is analyzed in Section 3.2 and some examples to show the feasibility of the proposal are shown in Section 4. Finally, the conclusions and future works are drafted in Section 5.

\section{Problem statement}

Let us consider the following class of uncertain nonlinear systems [13]:

$$
\left\{\begin{array}{l}
\dot{x}=A x+B_{u} u+B_{f} f(x, \omega(t), t), \\
y=C x
\end{array}\right.
$$

where $x=\left[x_{1}, \ldots, x_{n}\right]^{T} ; A \in \mathbb{R}^{n \times n} ; B_{u} \in \mathbb{R}^{n} ; B_{f} \in \mathbb{R}^{n}$; and $f(\cdot): \mathbb{R}^{n} \times \mathbb{R} \times \mathbb{R}_{\geq 0} \rightarrow \mathbb{R}$ is an uncertain possibly non-linear function. For the sake of simplicity, let us denote $f \triangleq f(x, \omega(t), t)$.

The traditional ESO is proposed for systems which are expressed in the CICF, that is, with

$$
\begin{aligned}
A & =\left[\begin{array}{ccccc}
0 & 1 & 0 & \cdots & 0 \\
0 & 0 & 1 & \cdots & 0 \\
\vdots & \vdots & \vdots & \ddots & \vdots \\
0 & 0 & 0 & \cdots & 1 \\
0 & 0 & 0 & \cdots & 0
\end{array}\right], \quad B_{u}=\left[\begin{array}{c}
0 \\
0 \\
\vdots \\
0 \\
b
\end{array}\right], \quad B_{f}=\left[\begin{array}{c}
0 \\
0 \\
\vdots \\
0 \\
1
\end{array}\right], \\
C & =\left[\begin{array}{ccccc}
1 & 0 & 0 & \cdots & 0
\end{array}\right] .
\end{aligned}
$$

On the other hand, the GESO deals with system (1) with arbitrary structure [13]. The control law proposed therein is given by

$$
u=K_{x} \hat{x}+k_{G} \hat{f}
$$

where

$$
k_{G}=-\left[C\left(A+B_{u} K_{x}\right)^{-1} B_{u}\right]^{-1} C\left(A+B_{u} K_{x}\right)^{-1} B_{f}
$$

is the disturbance compensation gain, $K_{x}$ is the feedback gain, and $\hat{x}, \hat{f}$ are estimations of $x, f$, respectively, which are obtained by constructing the following GESO:

$$
\left[\begin{array}{c}
\dot{\hat{x}} \\
\dot{\hat{x}}_{n+1}
\end{array}\right]=\left[\begin{array}{cc}
A & B_{f} \\
0_{1 \times n} & 0
\end{array}\right]\left[\begin{array}{c}
\hat{x} \\
\hat{x}_{n+1}
\end{array}\right]+\left[\begin{array}{c}
B_{u} \\
0
\end{array}\right] u+L(y-C \hat{x}),
$$

being, $L \in \mathbb{R}^{n+1}$ the observer gain and $\hat{x}_{n+1} \triangleq \hat{f}$.

Remark 1. The GESO is reduced to the traditional ESO when the matrices are given by (2).

Remark 2. If $K_{x}, L$ are chosen such that the system and the observer closed-loop matrices are Hurwitz, then, the bounded stability of (1) under (3)-(5) is guaranteed under the assumption of boundedness of $f$ and $\dot{f},[13]$.

In general, the control law (3)-(5) removes the effect of $f$ from the system output in steady state if $\lim _{t \rightarrow \infty} \dot{f}(t)=0$. In this paper, a further extension of (3) is proposed. First, an analysis is performed in order to show that, under perfect disturbance estimation, a dynamic disturbance rejection term can be designed such that $f$ is completely rejected from the system output without the need of imposing the steady-state requirement. Then, the closed-loop system stability when observation errors are considered is analyzed. 
Definition 1. The next definitions are made:

i). $\bar{A} \triangleq A+B_{u} K_{x}$.

ii). $z_{u, j} \in \mathbb{C}, j=1, \ldots, m_{u}$, with $z_{u, j} \neq z_{u, j+1}, \forall j$, denotes the zeros in the triplet $\left(\bar{A}, B_{u}, C\right)$.

iii). $n_{u, j} \in \mathbb{N}$ denotes the multiplicity of $z_{u, j}$.

iv). The zeros $z_{u, j}$, and their respective multiplicities $n_{u, j}$, are divided into: minimum phase zeros, $z_{u_{m}, j}, \quad n_{u_{m}, j}, j=1, \ldots, m_{u_{m}} ;$ non-minimum phase zeros, $z_{u_{n m}, j}, n_{u_{n m}, j}, j=1, \ldots, m_{u_{n m}} ;$ and zeros at the imaginary axis, $z_{u_{0}, j}, n_{u_{0}, j}, j=1, \ldots, m_{u_{0}} ;$ satisfying $m_{u}=m_{u_{m}}+m_{u_{n m}}+m_{u_{0}}$.

v). The total number of zeros in the triplet $\left(\bar{A}, B_{u}, C\right)$ is denoted by $m_{u}^{\text {tot }}=\sum_{i=1}^{m_{u}} n_{u, i}$. And, in the same way: $\quad m_{u_{m}}^{t o t}=\sum_{i=1}^{m_{u_{m}}} n_{u_{m}, i}, \quad m_{u_{n m}}^{t o t}=\sum_{i=1}^{m_{u_{n m}}} n_{u_{n m}, i}$ and $m_{u_{0}}^{\text {tot }}=\sum_{i=1}^{m_{u_{0}}} n_{u_{0}, i}$; are the total number of minimum phase zeros, non-minimum phase zeros and zeros at the imaginary axis, respectively.

vi). The same notation defined in ii), iii), iv) and v) is used for the triplet $\left(\bar{A}, B_{f}, C\right)$ by replacing the subindexes ' $u$ ' by ' $f$ '.

Also, the next assumptions are considered.

Assumption 1. The pair $\left(A, B_{u}\right)$ is controllable.

Assumption 2. The pair $(\bar{A}, C)$ is observable.

Assumption 1 is imposed for state-feedback design. Assumption 2 is imposed because, for the design of the disturbance rejection term, it is needed to compute $\mathcal{O}^{-1}$, being $\mathcal{O}$ the observability matrix of the pair $(\bar{A}, C)$.

\section{Proposed scheme}

The proposed control law has the following structure:

$$
\begin{aligned}
& u=K_{x} \hat{x}+u_{d}\left(\hat{\eta}, \hat{f}, \hat{\dot{f}}, \ldots, \hat{f}^{\left(k_{d}\right)}\right), \\
& \left\{\begin{array}{l}
\dot{\xi}=\Phi \xi+\Gamma \hat{f}, \\
\hat{\eta}=H \xi
\end{array}\right.
\end{aligned}
$$

where $K_{x}$ is chosen such that $\bar{A}$ is Hurwitz; $u_{d}(\cdot)$ is a function, subsequently derived, which is designed to reject the effect of $f$ from the system output; $k_{d}=\max \left\{0, m_{f}^{\text {tot }}-m_{u}^{\text {tot }}\right\}$; the matrices $\Phi \in \mathbb{R}^{p \times p}$ (Hurwitz), $\Gamma \in \mathbb{R}^{p}$ and $H \in \mathbb{R}^{1 \times p}, p=m_{u_{m}}^{\text {tot }}$, also subsequently defined, represent a dynamic component in the control law; and $\hat{x}, \hat{f}, \hat{\dot{f}}, \ldots, \hat{f}^{\left(k_{d}\right)}$, are estimations of $x, f, \dot{f}, \ldots, f^{\left(k_{d}\right)}$, respectively, which are obtained by constructing the next Extended States Observer:

$$
\dot{\hat{x}}_{e}=A_{e} \hat{x}_{e}+B_{e} u+L_{e}\left(y-C_{e} \hat{x}_{e}\right) ;
$$

where $L_{e}$ is the observer gain and

$$
\begin{aligned}
x_{e} & =\left[x, f, \dot{f}, \ldots, f^{\left(k_{d}\right)}\right]^{T}, \\
A_{e} & =\left[\begin{array}{ccc}
A & B_{f} & 0_{\left(n \times k_{d}\right)} \\
0_{\left(k_{d} \times n\right)} & 0_{\left(k_{d} \times 1\right)} & I_{\left(k_{d} \times k_{d}\right)} \\
0_{(1 \times n)} & 0_{(1 \times 1)} & 0_{\left(1 \times k_{d}\right)}
\end{array}\right], B_{e}=\left[\begin{array}{c}
B_{u} \\
0_{\left(k_{d} \times 1\right)} \\
0_{(1 \times 1)}
\end{array}\right], \\
C_{e} & =\left[\begin{array}{lll}
C & 0_{(1 \times 1)} & 0_{\left(1 \times k_{d}\right)}
\end{array}\right] .
\end{aligned}
$$

The next section is devoted to find the explicit expression of $u_{d}(\cdot)$ and the matrices $\Phi, \Gamma, H$; then, once $u_{d}(\cdot), \Phi, \Gamma, H$ are determined, the closed-loop system stability is analyzed in Section 3.2.

\subsection{Rejection of the Mismatched Uncertainty}

In order to determine $u_{d}(\cdot)$, let us consider that $\hat{x}=x$, $\hat{f}=f, \hat{\dot{f}}=\dot{f}, \ldots, \hat{f}^{\left(k_{d}\right)}=f^{\left(k_{d}\right)}$ and $\hat{\eta}=\eta$. In this ideal scenario, the closed-loop system formed by $(1),(6)$ is

$$
\left\{\begin{array}{l}
\dot{x}=\bar{A} x+B_{u} u_{d}(\cdot)+B_{f} f, \\
y=C x
\end{array}\right.
$$

Now, let us show how $u_{d}(\cdot)$ is unequivocally determined if it is designed to reject the effect of $f$ from the system output. The next proposition is needed for the subsequent analysis.

Proposition 1. Under Assumption 2, the system (9) is input-output equivalent to

$$
\left\{\begin{aligned}
\dot{v}_{1} & =v_{2} \\
\dot{v}_{2} & =v_{3} \\
\vdots & \\
\dot{v}_{n} & =C \bar{A}^{n} \mathcal{O}^{-1} v+b_{u} \bar{u}_{d}+b_{f} \bar{f} \\
y & =v_{1} .
\end{aligned}\right.
$$

being $\bar{u}_{d} \triangleq\left[u_{d}, \dot{u}_{d}, \cdots, u_{d}^{(n-1)}\right]^{T}, \quad \bar{f} \triangleq\left[f, \dot{f}, \cdots, f^{(n-1)}\right]^{T}$, $\mathcal{O}$ the observability matrix of system $(9), v_{i}=y^{(i-1)}$, $v=\left[v_{1}, \ldots, v_{n}\right]^{T}$ and

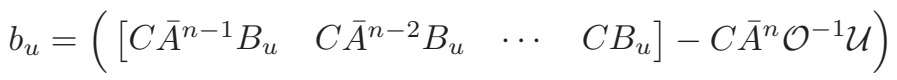

$$
\begin{aligned}
& \triangleq\left[\begin{array}{llll}
b_{u, 0} & b_{u, 1} & \cdots & b_{u, n-1}
\end{array}\right],
\end{aligned}
$$

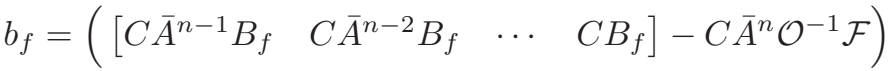

$$
\begin{aligned}
& \triangleq\left[\begin{array}{llll}
b_{f, 0} & b_{f, 1} & \cdots & b_{f, n-1}
\end{array}\right] \text {. }
\end{aligned}
$$

with

$$
\begin{aligned}
\mathcal{U} & =\left[\begin{array}{ccccc}
0 & 0 & \ldots & 0 & 0 \\
C B_{u} & 0 & \ldots & 0 & 0 \\
C \bar{A} B_{u} & C B_{u} & \ldots & 0 & 0 \\
\vdots & \vdots & \ddots & \vdots & \vdots \\
C \bar{A}^{n-2} B_{u} & C \bar{A}^{n-3} B_{u} & \ldots & C B_{u} & 0
\end{array}\right] \\
\mathcal{F} & =\left[\begin{array}{ccccc}
0 & 0 & \ldots & 0 & 0 \\
C B_{f} & 0 & \ldots & 0 & 0 \\
C \bar{A} B_{f} & C B_{f} & \ldots & 0 & 0 \\
\vdots & \vdots & \ddots & \vdots & \vdots \\
C \bar{A}^{n-2} B_{f} & C \bar{A}^{n-3} B_{f} & \ldots & C B_{f} & 0
\end{array}\right]
\end{aligned}
$$

Proof. See Appendix A.

The representation (10)-(11) has two remarkable properties, which are indicated in the following lemma: 
Lemma 1. The coefficients $b_{u}$ and $b_{f}$ in (11) satisfy:

1. $b_{u, i}=0, \forall i>m_{u}^{\text {tot }}$ and $b_{f, i}=0, \forall i>m_{f}^{\text {tot }}$.

2. The roots of $b_{u, 0}+b_{u, 1} s+\ldots+b_{u, n-1} s^{n-1}$ and $b_{f, 0}+b_{f, 1} s+\ldots+b_{f, n-1} s^{n-1}$, are equal to the zeros $z_{u, j}, j=1, \ldots, m_{u}$ and $z_{f, j}, j=1, \ldots, m_{f}$; respectively.

Proof. See Appendix B.

Proposition 1 shows that, in order to remove the effect of $f$ from the system output, it is needed to introduce a disturbance rejection term, $u_{d}$, which solves the differential equation $b_{u} \bar{u}_{d}+b_{f} \bar{f}=0$. This is developed in Theorem 1 and Corollary 1.

Theorem 1. If $\left(\bar{A}, B_{u}, C\right)$ does not have non-minimum phase zeros $\left(m_{u_{n m}}=0\right)$, then the effect of $f$ is rejected from the system output if, and only if,

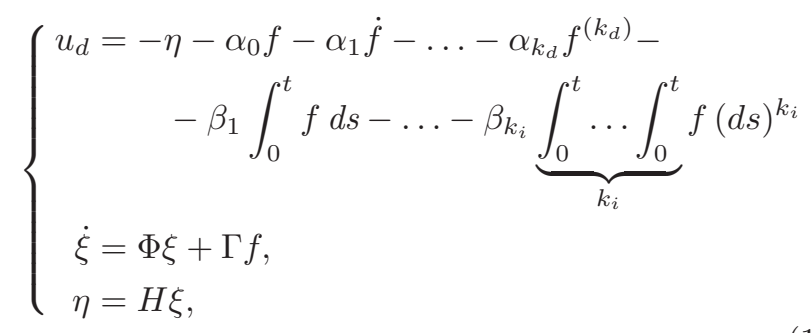

where $\alpha_{0}, \alpha_{1}, \ldots, \alpha_{k_{d}} \in \mathbb{R}, k_{d}=\max \left\{0, m_{f}^{\text {tot }}-m_{u}^{\text {tot }}\right\}$, are given by $(21) ; \beta_{1}, \ldots, \beta_{k_{i}} \in \mathbb{R}, k_{i}=\max \left\{0, m_{u_{0}}^{\text {tot }}-m_{f_{0}}^{\text {tot }}\right\}$, are given by (20), and the matrices $\Phi \in \mathbb{C}^{p \times p}, \Gamma \in \mathbb{C}^{p \times 1}$, $H \in \mathbb{C}^{1 \times p}, p=m_{u_{m}}^{\text {tot }}$, being $\Phi$ Hurwitz, are determined by

$$
\begin{aligned}
\Phi & =\left[\begin{array}{cccc}
\Phi_{1} & 0 & \ldots & 0 \\
0 & \Phi_{2} & \ldots & 0 \\
\vdots & \vdots & \ddots & \vdots \\
0 & 0 & \ldots & \Phi_{m_{u_{m}}}
\end{array}\right], \quad \Gamma=\left[\begin{array}{c}
\Gamma_{1} \\
\Gamma_{2} \\
\vdots \\
\Gamma_{m_{u_{m}}}
\end{array}\right], \\
H & =\left[\begin{array}{llll}
H_{1} & H_{2} & \ldots & H_{m_{u_{m}}}
\end{array}\right],
\end{aligned}
$$

where $\Phi_{j} \in \mathbb{C}^{\left(n_{u_{m}, j}\right) \times\left(n_{u_{m}, j}\right)}, \quad \Gamma_{j} \in \mathbb{C}^{\left(n_{u_{m}, j}\right) \times 1}$, $H_{j} \in \mathbb{C}^{1 \times\left(n_{u_{m}, j}\right)}$ are given by

$$
\begin{aligned}
& \Phi_{j}=\left[\begin{array}{ccccc}
z_{u_{m}, j} & 1 & 0 & \ldots & 0 \\
0 & z_{u_{m}, j} & 1 & \ldots & 0 \\
0 & 0 & z_{u_{m}, j} & \ldots & 0 \\
\vdots & \vdots & \vdots & \ddots & \vdots \\
0 & 0 & 0 & \ldots & z_{u_{m}, j}
\end{array}\right], \quad \Gamma_{j}=\left[\begin{array}{c}
0 \\
0 \\
\vdots \\
1
\end{array}\right] \\
& H_{j}=\left[\begin{array}{llll}
r_{g_{s}}^{j,\left(n_{u_{m}, j}\right)} & r_{g_{s}}^{j,\left(n_{u_{m}, j}\right)-1} & \ldots & r_{g_{s}}^{j, 1}
\end{array}\right],
\end{aligned}
$$

with the coefficients $r_{g_{s}}^{j, k} \in \mathbb{C}$, with $j=1, \ldots, m_{u_{m}}$, $k=1, \ldots, n_{u_{m}, j}$ determined by (18).

Proof. From (10) it is concluded that if $u_{d}$ is chosen such that the following differential equation holds

$$
b_{u} \bar{u}_{d}+b_{f} \bar{f}=0,
$$

then the effect of $f$ is removed from the system output. As (15) is linear in $u_{d}$ and $f$, the Laplace transform can be used to solve it. Applying the Laplace transform to (15) leads to

$$
U_{d}(s)=-\frac{b_{f, 0}+b_{f, 1} s+\ldots+b_{f, n-1} s^{(n-1)}}{b_{u, 0}+b_{u, 1} s+\ldots+b_{u, n-1} s^{(n-1)}} F(s) .
$$

By Lemma 1 it is known that the roots of the numerator and the denominator in (16) are the zeros of $\left(\bar{A}, B_{f}, C\right)$ and $\left(\bar{A}, B_{u}, C\right)$, respectively. Therefore, by means of the partial fraction expansion, equation (16) can be always decomposed as

$$
U_{d}(s)=-\left(G_{s}(s)+G_{u}(s)+p_{d}(s)+p_{i}(1 / s)\right) F(s) .
$$

with

$$
\begin{aligned}
& G_{s}(s)=\sum_{j=1}^{m_{u_{m}}}\left(\sum_{k=1}^{n_{u_{m}, j}} \frac{r_{g_{s}}^{j, k}}{\left(s-z_{u_{m}, j}\right)^{k}}\right), \quad \operatorname{Re}\left\{z_{u_{m}, j}\right\}<0 \\
& G_{u}(s)=\sum_{j=1}^{m_{u_{n} m}}\left(\sum_{k=1}^{n_{u_{n m}, j}} \frac{r_{g_{u}}^{j, k}}{\left(s-z_{u_{n m}, j}\right)^{k}}\right), \operatorname{Re}\left\{z_{u_{n m}, j}\right\}>0 \\
& p_{i}(1 / s)=\sum_{j=1}^{k_{i}} \beta_{j}\left(\frac{1}{s}\right)^{j}, \quad k_{i}=\max \left\{0, m_{u_{0}}^{\text {tot }}-m_{f_{0}}^{\text {tot }}\right\} \\
& p_{d}(s)=\sum_{j=0}^{k_{d}} \alpha_{j} s^{j}, \\
& k_{d}=\max \left\{0, m_{f}^{\text {tot }}-m_{u}^{\text {tot }}\right\}
\end{aligned}
$$

with $r_{g_{s}}^{j, k}, r_{g_{u}}^{j, k} \in \mathbb{C}$ and $\alpha_{j}, \beta_{j} \in \mathbb{R}$.

The proof follows by imposing the condition of the absence of non-minimum phase zeros, $m_{u_{n m}}=0$, which forces $G_{u}(s)=0$. Therefore, if the inverse Laplace transform is applied to (17)-(21), it leads to (12)-(14), where (13)-(14) is the Jordan canonical form of (18).

Remark 3. Note that, by Theorem 1, the explicit form of the disturbance rejection term, $u_{d}(\cdot)$, is exclusively determined by the zeros of the system. This illustrates that the internal zero-dynamics plays an important role in ESObased control design.

Remark 4. The coefficients $\alpha_{j}, \beta_{j}$ in (12), and $r_{g_{s}}^{j, k}$ in (14), need to be computed in order to implement the control law. Those coefficients can be directly obtained by a wide number of mathematical tools, e.g. by the command residue $\left(b_{f}, b_{u}\right)$ in MatLab ${ }^{\circledR}$.

Remark 5. The disturbance rejection term, $u_{d}(\cdot)$, is reduced to the one in the GESO-based control by imposing the steady-state in the differential equation (15). In this case, $u_{d}\left(\eta, f, \ldots, f^{\left(k_{d}\right)}\right)=k_{d} f$, where $k_{d}=-b_{f, 0} / b_{u, 0}$. Also, it can be easily verified that, if the matrices are given by (2), then (12) leads to the conventional ESO-based control law $u_{d}(t)=-b f$. 
In Theorem 1 it is necessary to impose the condition $m_{u_{n m}}=0$. Otherwise, when applying the inverse Laplace transform, the matrix $\Phi$ in (12) would be the state space realization of $G_{s}(s)+G_{u}(s)$ containing unstable poles. The next Corollary establishes a result when $m_{u_{n m}} \neq 0$.

Corollary 1. If $\left(\bar{A}, B_{u}, C\right)$ has non-minimum phase zeros $\left(m_{u_{n m}} \neq 0\right)$, then the effect of $f$ is attenuated from the system output if its maximum frequency, $\omega_{f}^{\max }(\mathrm{rad} / \mathrm{s})$, satisfies that $\left\|\omega_{f}\right\|<\min _{j}\left\{\operatorname{Re}\left\{z_{u_{n m}, j}\right\}\right\}$ and

$$
\left\{\begin{aligned}
u_{d} & =-\eta-\alpha_{0} f-\ldots-\alpha_{k_{d}} f^{\left(k_{d}\right)}-\gamma_{0} f-\ldots-\gamma_{k_{d}} f^{\left(k_{d}\right)} \\
& -\beta_{1} \int_{0}^{t} f d s-\ldots-\beta_{k_{i}} \underbrace{\int_{0}^{t} \ldots \int_{0}^{t}}_{k_{i}} f(d s)^{k_{i}} \\
\dot{\xi} & =\Phi \xi+\Gamma f \\
\eta & =H \xi
\end{aligned}\right.
$$

where the new coefficients $\gamma_{0}, \gamma_{1}, \ldots, \gamma_{k_{d}} \in \mathbb{R}$, are given by $(23)$.

Proof. From the proof of Theorem 1, if $G_{u}(s) \neq 0$ then, by means of the Taylor expansion around $s=0, G_{u}(s)$ can be approximated by a $k_{d}$-order polynomial:

$$
G_{u}(s) \approx \sum_{j=0}^{k_{d}} \gamma_{j} s^{j} \triangleq p_{u}(s), \quad \forall\|s\|<\min _{j}\left\{\operatorname{Re}\left\{z_{u_{n m}, j}\right\}\right\} .
$$

As this Taylor expansion is only defined when $\|s\|<\min _{j}\left\{\operatorname{Re}\left\{z_{u_{n m}, j}\right\}\right\}$, then frequencies higher than $\min _{j}\left\{\operatorname{Re}\left\{z_{u_{n m}, j}\right\}\right\}$ in the disturbance may cause disturbance amplification.

Remark 6. It can be seen that (12) and (22) match with the definition $u_{d}\left(\eta, f, \dot{f}, \ldots, f^{\left(k_{d}\right)}\right)$ in (6).

Remark 7. The integrals appearing in (12), (22) may cause confusion as it may be thought that, in general, constant uncertainties will cause unbounded control actions. However, the theorem states that the integrals only appear when $m_{u_{0}}^{\text {tot }}-m_{f_{0}}^{\text {tot }}>0$, and these are very singular systems which rarely appear in practice. For this reason, this case will not be considered in the sequel.

\subsection{Closed-loop stability}

The closed-loop stability is analyzed when system (1) is controlled by (6). The following assumptions are imposed.

Assumption 3. The pair $\left(A_{e}, C_{e}\right)$ is observable.

Assumption 4. The triplet $\left(\bar{A}, B_{u}, C\right)$ does not have zeros at the imaginary axis.

Assumption 5. $f \triangleq f(x, \omega(t), t) \equiv \bar{f}(\omega(t), t),[13]$

Assumption 6. $f^{\left(k_{d}+1\right)}$ is bounded.
Assumption 3 is imposed for observer pole-placement. Assumption 4 rules out the case pointed out in Remark 7 in which the system only responds to the rate of variation of the control input rather than its actual value. It is considered in order to simplify the stability analysis as it forces that no integrals appear in $u_{d}(\cdot)$. Assumptions 5-6 are widely employed in the ESO-based control literature $[12,13,25,32]$. In fact, dealing directly with $f(x, \omega(t), t)$ without assuming boundedness led to important technical difficulties, being an actual challenging topic [1, 10]. These assumptions, are reasonable when the system stability is not affected by the lumped disturbance. In that case, $x(t)$ in $f$ is interpreted as an external time-varying function (in the same way as $\omega(t)$ ) and $f(x, \omega(t), t) \equiv \bar{f}(\omega(t), t)$. This happens in many control applications whose major dynamics are stabilized by feedback and the resulting lumped disturbance term is weak enough so that the stability is not affected [13].

Now, let us define the observation error as $e_{o} \triangleq x_{e}-\hat{x}_{e}$, with $x_{e}$ defined in (8); and $e_{\eta} \triangleq \eta-\hat{\eta}$. The following lemma proves that these errors are bounded.

Lemma 2. Under Assumptions 3, 5, 6, the boundedness of $e_{o}$ and $e_{\eta}$ is guaranteed if $L_{e}$ in $(7)$ is chosen such that $A_{e}-L_{e} C_{e}$ is Hurwitz.

Proof. First, let us prove that $e_{o}$ is bounded. Rewrite (1) as

$$
\left\{\begin{array}{l}
\dot{x}=A x+B_{f} x_{f, 0}+B_{u} u \\
\dot{x}_{f, 0}=x_{f, 1} \\
\vdots \\
\dot{x}_{f, k_{d}}=f^{\left(k_{d}+1\right)}
\end{array}\right.
$$

where $\left[x_{f, 0}, x_{f, 1}, \ldots, x_{f, k_{d}}\right]^{T}=\left[f, \dot{f}, \ldots, f^{\left(k_{d}\right)}\right]^{T} . \quad \mathrm{Ex}-$ pressing (24) in matrix form lead to

$$
\dot{x}_{e}=A_{e} x_{e}+B_{e} u+B_{e, f} f^{\left(k_{d}+1\right)}
$$

with $x_{e}, A_{e}, B_{e}$ defined in (8) and $B_{e, f}=\left[0_{1 \times\left(n+k_{d}\right)}, 1\right]^{T}$.

By differentiating $e_{o} \triangleq x_{e}-\hat{x}_{e}$ and incorporating (24) and $(7)$ it is concluded that

$$
\dot{e}_{o}=\left(A_{e}-L_{e} C_{e}\right) e_{o}+B_{e, f} f^{\left(k_{d}+1\right)}
$$

which is bounded, for any $f^{\left(k_{d}+1\right)}$ bounded, since $\left(A_{e}-L_{e} C_{e}\right)$ is Hurwitz [25].

Let us now show that $e_{\eta}$ is bounded. As $e_{o}$ is bounded, it implies that $e_{f} \triangleq f-\hat{f}$ is also bounded. From (6), one has that

$$
\left\{\begin{array}{l}
\dot{\xi}=\Phi \xi+\Gamma \hat{f}=\Phi \xi+\Gamma\left(f-e_{f}\right), \\
\hat{\eta}=H \xi
\end{array}\right.
$$

then, by the superposition principle, $\hat{\eta}=\eta+e_{\eta}$, where $e_{\eta}$ is given by

$$
\left\{\begin{array}{l}
\dot{\xi}=\Phi \xi-\Gamma e_{f}, \\
e_{\eta}=H \xi
\end{array}\right.
$$


As $\Phi$ is Hurwitz, and $e_{f}$ is bounded, then $e_{\eta}$ is also bounded.

Now, the next lemma expresses the control action $u$, defined in (6), in terms of the actual states, $x$, and the actual disturbances, $\eta, f, \dot{f}, \ldots, f^{\left(k_{d}\right)}$, plus some terms depending on the previously defined errors.

Lemma 3. Suppose Assumption 4 is satisfied. Then, control law (6) is

$$
u=K_{x} x+u_{d}\left(\eta, f, \ldots, f^{\left(k_{d}\right)}\right)-\left[K_{x},-\lambda\right] e_{o}-e_{\eta},
$$

where $\lambda=\left[\lambda_{0}, \ldots, \lambda_{k_{d}}\right]$, with $\lambda_{i}=\alpha_{i}+\gamma_{i}, i=0, \ldots, k_{d}$.

Proof. It is verified that

$$
u=K_{x} \hat{x}-\hat{\eta}-\lambda_{0} \hat{f}-\ldots-\lambda_{k} \hat{f}^{\left(k_{d}\right)}=\left[K_{x}-\lambda\right] \hat{x}_{e}-\hat{\eta},
$$

and that

$$
\begin{aligned}
K_{x} x+u_{d}\left(\eta, f, \ldots, f^{\left(k_{d}\right)}\right) & =K_{x} x-\eta-\lambda_{0} f-\ldots-\lambda_{k} f^{\left(k_{d}\right)} \\
& =\left[K_{x}-\lambda\right] x_{e}-\eta .
\end{aligned}
$$

Then, the proof immediately follows by subtracting both expressions.

Finally, the next theorem establishes the bounded stability of $y(t)$.

Theorem 2. Suppose Assumptions 1-6 are satisfied, then the bounded stability of $y(t)$, when system (1) is being controlled with (6)-(7) is guaranteed if $L_{e}$ is chosen such that $A_{e}-L_{e} C_{e}$ is Hurwitz and $K_{x}$ is chosen such that $\bar{A}$ is Hurwitz.

Proof. The closed-loop system (1), (27) is

$$
\left\{\begin{array}{l}
\dot{x}=\bar{A} x+B_{u} u_{d}\left(\eta, f, \ldots, f^{\left(k_{d}\right)}\right)+B_{f} f- \\
\quad-B_{u}\left[K_{x},-\lambda\right] e_{o}-B_{u} e_{\eta}, \\
y=C x,
\end{array}\right.
$$

which proves that $y(t)$ is stable since $B_{u} u_{d}\left(\eta, f, \ldots, f^{\left(k_{d}\right)}\right)$ exactly rejects the effect of $B_{f} f$ in the system output (it has been designed for that purpose in section 3.1), and $e_{o}, e_{\eta}$ are bounded by Lemma 2 .

Theorem 2 proves that the system output is bounded for any kind of disturbance satisfying that $f^{\left(k_{d}+1\right)}$ is bounded. However, it is important to note that the disturbance attenuation capabilities may be better, in general, for lowfrequency disturbances rather than for high-frequency disturbances. This fact can be easily seen in equation (26), which shows that the observation error produced by the observer (7)-(8) is driven by $f^{\left(k_{d}+1\right)}$. Therefore, highfrequencies, which imply $\left\|f^{\left(k_{d}+1\right)}\right\|>>0$, may cause nonnegligible observation errors, degrading the disturbance rejection capabilities of (12) or (22).

However, it is also important to realize that equation (12) constitutes a sufficient, and necessary, condition in order to reject the mismatched uncertainty. Therefore, even under perfect observation conditions, rejecting highfrequency disturbances may imply that high control actions are needed, and the maximum value of $u(t)$ is limited in practical applications. So, if high-frequency mismatched disturbances are going to affect the system, then, depending on each particular case, it may be more convenient to implement an approximation of the control law (12), (22) by eliminating some of the higher derivatives.

\section{Numerical examples}

In this section some comparative examples are considered to illustrate the main ideas of the paper. In Example 1 a comparison with respect to the GESO is considered. In Example 2, the proposed control law is applied to an uncertain unstable system with an internal minimum-phase zero. It is compared with the GESO and also, for the sake of completeness, the system is also simulated under the conventional ESO when the internal zero is neglected. Finally, Example 3 is introduced to show the feasibility of the proposal when attenuating periodical signals in nonminimum phase systems.

Example 1. Let us consider the system presented in [13]:

$$
\left\{\begin{aligned}
\dot{x}_{1} & =x_{2}+e^{x_{1}}+\omega(t) \\
\dot{x}_{2} & =-2 x_{1}-x_{2}+u(t) \\
y & =x_{1}
\end{aligned}\right.
$$

being $f(x, \omega(t), t) \triangleq e^{x_{1}}+\omega(t)$, with $\omega(t)=0,0 \leq t<5$ and $\omega(t)=3, t \geq 5$. The system matrices are:

$$
\begin{aligned}
& A=\left[\begin{array}{cc}
0 & 1 \\
-2 & -1
\end{array}\right], \quad B_{u}=\left[\begin{array}{l}
0 \\
1
\end{array}\right], \quad B_{f}=\left[\begin{array}{l}
1 \\
0
\end{array}\right] \\
& C=\left[\begin{array}{ll}
1 & 0
\end{array}\right] .
\end{aligned}
$$

The feedback control law (6) is set with $K_{x}=[-4,-4]$ so that $\bar{A}=A+B_{u} K_{x}$ is

$$
\bar{A}=\left[\begin{array}{cc}
0 & 1 \\
-6 & -5
\end{array}\right]
$$

with poles at $p=[-2,-3]^{T}$. The coefficients $b_{u}, b_{f}$ are computed by (11) resulting in $b_{u}=\left[b_{u, 0}, b_{u, 1}\right]=[1,0]$, $b_{f}=\left[b_{f, 0}, b_{f, 1}\right]=[5,1] . \quad$ By Lemma 1 it is known that the zeros of $\left(\bar{A}, B_{u}, C\right)$ and $\left(\bar{A}, B_{f}, C\right)$ are the roots of $b_{u, 0}+b_{u, 1} s=1$ and $b_{f, 0}+b_{f, 1} s=5+s$, respectively. Hence, $\quad m_{u}^{t o t}=m_{u_{0}}^{t o t}=m_{u_{m}}^{t o t}=m_{u_{n m}}^{\text {tot }}=0$; $m_{f}^{\text {tot }}=m_{f_{m}}^{\text {tot }}=1$; and $m_{f_{0}}^{\text {tot }}=m_{f_{n m}}^{\text {tot }}=0$. As the system does not have non-minimum phase zeros, the term $u_{d}(\cdot)$ in the control law (6) is obtained by direct application of Theorem 1 with $k_{d}=1, k_{i}=0, p=0$, leading to:

$$
u_{d}(f, \dot{f})=-\alpha_{0} f-\alpha_{1} \dot{f} .
$$

where the coefficients $\alpha_{0}=5, \alpha_{1}=1$ are given by (21). Therefore, control law (6) results in

$$
u=K_{x} \hat{x}-5 \hat{f}-\hat{\dot{f}}
$$



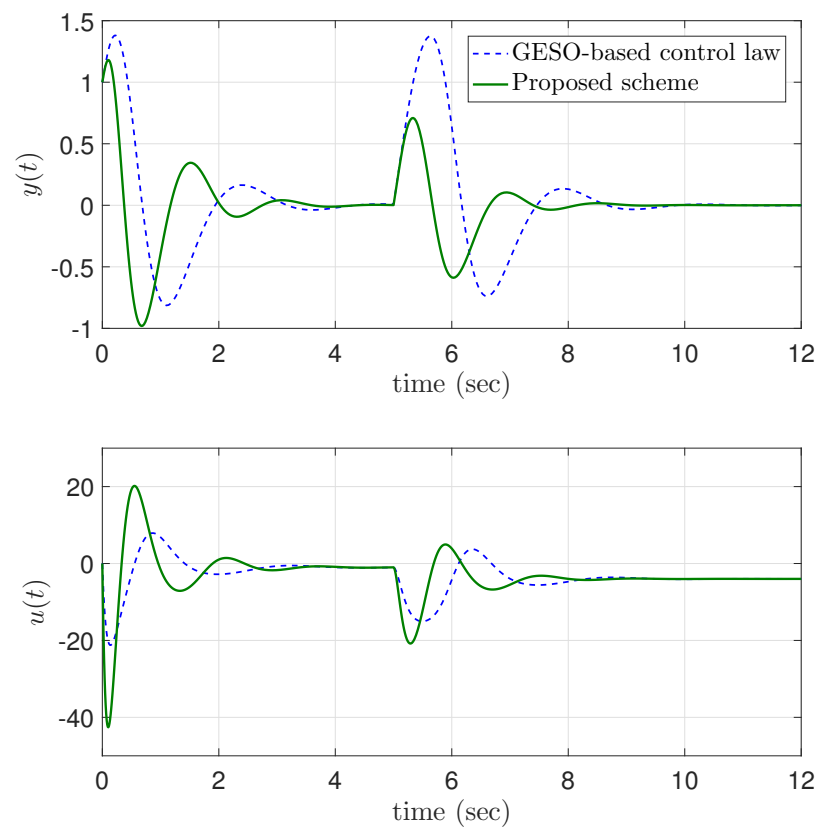

Figure 1: Simulation of Example 1.

where the estimations $\hat{x}, \hat{f}, \hat{\dot{f}}$ are obtained by the ESO with $k_{d}=1$.

In order to establish a comparison, another simulation is performed with the GESO-based control law (3), which results in

$$
u=K_{x} \hat{x}+k_{G} \hat{f}=K_{x} \hat{x}-5 \hat{f}
$$

where $k_{G}$ is given by (4) and the estimations are obtained by (5).

Simulation results can be seen in Figure 1. The observer gains has been set in order to place the observer poles at $s=-5$. The system initial state is $x_{0}=[1,0]^{T}$ while the observer initial state has been set to zero. The GESObased control law produces an Integral Amplitude Error (IAE) of 3.12, while with this proposal the IAE is reduced in a $56 \%(\mathrm{IAE}=1.76)$.

Example 2. Let us now consider the following unstable system whose nominal part has an internal minimumphase zero:

$$
\left\{\begin{aligned}
\dot{x}_{1} & =3 x_{1}-1.5 x_{2}+0.5 x_{3}+2 u(t), \\
\dot{x}_{2} & =2 x_{1} \\
\dot{x}_{3} & =x_{2}+\tanh \left(x_{3}\right)+\omega(t) \\
y & =0.25 x_{2}+0.75 x_{3} .
\end{aligned}\right.
$$

with $f(x, \omega(t)) \triangleq \tanh \left(x_{3}\right)+\omega(t) ; \omega(t)=0,0 \leq t<5$ and $\omega(t)=5 \bar{t} e^{-\frac{1}{2} \bar{t}}, t \geq 5$ being $\bar{t}=t-5$, as depicted in Figure 2. The system matrices are

$$
\begin{aligned}
A & =\left[\begin{array}{ccc}
3 & -1.5 & 0.5 \\
2 & 0 & 0 \\
0 & 1 & 0
\end{array}\right], \quad B_{u}=\left[\begin{array}{l}
2 \\
0 \\
0
\end{array}\right], \quad B_{f}=\left[\begin{array}{l}
0 \\
0 \\
1
\end{array}\right] \\
C & =\left[\begin{array}{lll}
0 & 0.25 & 0.75
\end{array}\right] .
\end{aligned}
$$
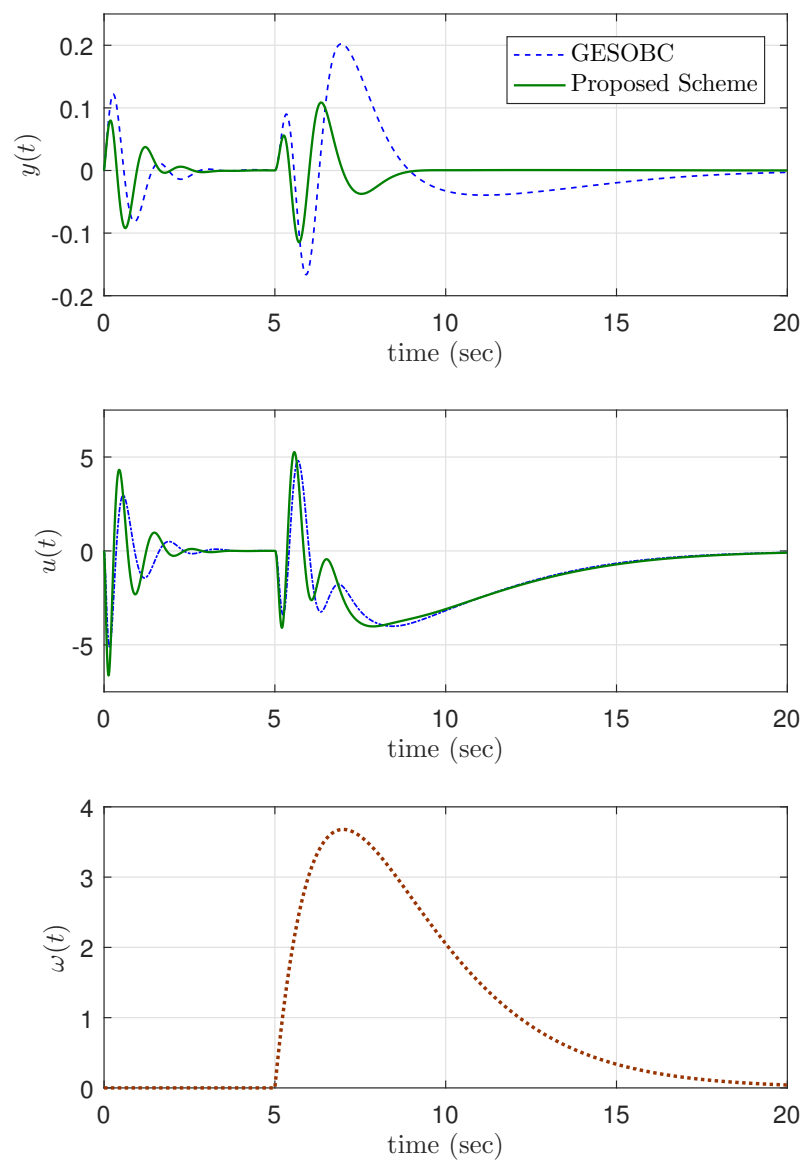

Figure 2: Simulation of Example 2.

being $\operatorname{det}(\lambda I-A)=(\lambda-1)^{3}$.

In what follows, the proposed control law, the GESObased control law and the conventional ESO are developed for system (31).

Proposed scheme: The feedback gain is set to $K_{x}=[9,18,31.5]$ so that the the matrix $\bar{A}$ becomes:

$$
\bar{A}=\left[\begin{array}{ccc}
-15 & -37.5 & -62.5 \\
2 & 0 & 0 \\
0 & 1 & 0
\end{array}\right] .
$$

with poles at $s=-5$.

The coefficients $b_{u}, b_{f}$ are computed by (11) leading to $b_{u}=[3,1,0]$ and $b_{f}=[25,11.25,0.75]$. Hence, by Lemma 1 the zeros of $\left(\bar{A}, B_{u}, C\right)$ and $\left(\bar{A}, B_{f}, C\right)$ are given by the roots of $(3+s)$ and $\left(25+11.25 s+0.75 s^{2}\right)$, respectively. Then, $m_{u}^{t o t}=m_{u_{m}}^{t o t}=m_{u_{m}}=1$ and $z_{u_{m}, 1}=-3$, $n_{z_{u_{m}, 1}}=1$; while $m_{f}^{\text {tot }}=m_{f_{m}}^{\text {tot }}=m_{f_{m}}=2$ and $z_{f_{m}, 1}=-12.287, n_{z_{f_{m}, 1}}=1, z_{f_{m}, 2}=-2.713, n_{z_{f_{m}, 2}}=1$.

As the triplet $\left(\bar{A}, B_{u}, C\right)$ does not have non-minimum phase zeros, then $u_{d}(\cdot)$ is determined by direct application of Theorem 1 with $k_{d}=1, k_{i}=0, p=1$, resulting in

$$
\left\{\begin{array}{l}
u_{d}(\eta, f, \dot{f}, \ddot{f})=-\eta-\alpha_{0} f-\alpha_{1} \dot{f}, \\
\dot{\xi}=\Phi_{1} \xi+\Gamma_{1} f \\
\eta=H_{1} \xi
\end{array}\right.
$$



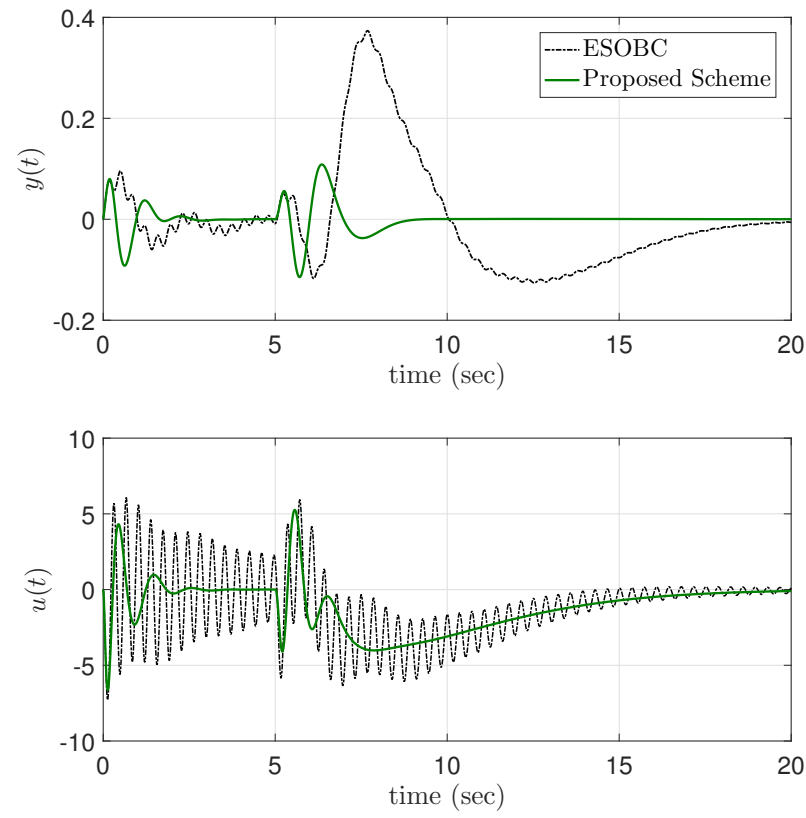

Figure 3: Simulation of Example 2 when the traditional ESO is applied neglecting the internal zero.

with $\alpha_{0}=9, \alpha_{1}=0.75$, determined by (21); and $\Phi_{o}=-3$, $\Gamma_{o}=1$ and $H_{o}=-2$, determined by (13)-(14) with $r_{g_{s}}^{1,1}$ given by (18). Then the resulting control law is given by (6) where the estimations are obtained by (7) with $k_{d}=1$.

GESO-based control: The matrix $K_{x}$ is set with same values. Therefore, the control law is given by

$$
u=K_{x} \hat{x}+k_{G} \hat{f}=K_{x} \hat{x}-8.33 \hat{f}
$$

where $k_{G}$ is given by (4) and the estimations are obtained by $(5)$.

The simulation is presented in Figure 2, where the proposed control law and the GESO-based control law are introduced into (31). The system initial state is $x_{0}=[1,0,0]^{T}$ and the observer gains has been set to place the observer poles at $s=-5$. It can be seen how the GESO rejects the disturbance when the steady-state is reached, while with the proposed scheme the disturbance is rejected before, i.e. when it is satisfied that $\hat{f} \approx f$, $\hat{\dot{f}} \approx \dot{f}$.

Conventional ESO-based control: For the sake of completeness, let us show what would happen if the traditional ESO is designed for system (31) neglecting the presence of the internal zero. First, the system is expressed in cascade form:

$$
\left\{\begin{array}{l}
\dot{v}_{1}=v_{2}, \\
\dot{v}_{2}=v_{3}, \\
\dot{v}_{3}=\omega(v, f, \dot{u})+3 u \\
y=v_{1}
\end{array}\right.
$$

where

$$
\omega(f(t), \dot{u})=v_{1}-3 v_{2}+3 v_{3}+\dot{u}+2.5 f-2.25 \dot{f}+0.75 \ddot{f}
$$

is considered as the lumped disturbance.

The feedback matrix is set to $K_{v}=[-41.7,-25,-5]$ so that the closed-loop poles of the nominal part of (33) are placed at $s=-5$. An extended state $v_{4}=\omega(\cdot)$ is considered so that the ESO control law results in

$$
u(t)=K_{v} \hat{v}-\frac{\hat{v}_{4}}{3},
$$

where the estimations are obtained by constructing an ESO for system (33) with poles at $s=-5$.

A simulation is depicted in Figure 3 where it can be seen that the conventional ESO-based control produces oscillations in the control action due to the presence of $\dot{u}$ in the lumped uncertainty. If the observer gains are increased, it becomes more unstable. Instability appear when the observer poles are placed at $s=-6$. This shows that the knowledge of the internal zero-dynamics is crucial in ESO-based control design.

Example 3. Let us change system (31) by the following one having an internal non-minimum phase zero:

$$
\left\{\begin{aligned}
\dot{x}_{1} & =-3 x_{1}-1.5 x_{2}-0.5 x_{3}+u(t) \\
\dot{x}_{2} & =2 x_{1} \\
\dot{x}_{3} & =x_{2}+\sin \left(\frac{\pi}{5} t\right)+\sin \left(x_{3}\right) \cos \left(x_{2}\right) \\
y & =0.5 x_{1}-x_{2}
\end{aligned}\right.
$$

whose new matrices are

$$
\begin{aligned}
A & =\left[\begin{array}{ccc}
-3 & -1.5 & -0.5 \\
2 & 0 & 0 \\
0 & 1 & 0
\end{array}\right], \quad B_{u}=\left[\begin{array}{l}
1 \\
0 \\
0
\end{array}\right], \quad B_{f}=\left[\begin{array}{l}
0 \\
0 \\
1
\end{array}\right] \\
C & =\left[\begin{array}{lll}
0.5 & -1 & 0
\end{array}\right] .
\end{aligned}
$$

If $x(0)=0$ and $u(t)=0$, then this system is stable for all $t=[0, \infty)$. Therefore, $K_{x}$ is set to zero and just the performance of $u(t)=u_{d}(t)$ in attenuating $f(x, t)=\sin \left(\frac{\pi}{5} t\right)+\sin \left(x_{3}\right) \cos \left(x_{2}\right)$ will be evaluated.

The coefficients $b_{u}, b_{f}$ are computed by (11) leading to $b_{u}=[-2,1,0]$ and $b_{f}=[-3.5,-3,-1]$. Hence, by Lemma 1 the zeros of $\left(A, B_{u}, C\right)$ and $\left(A, B_{f}, C\right)$ are given by the roots of $(-2+s)$ and $\left(-3.5-3 s-s^{2}\right)$, respectively. So, $m_{u}^{\text {tot }}=m_{u_{n m}}^{\text {tot }}=m_{u_{n m}}=1$. As $m_{u_{n m}} \neq 0$, the results of Corollary 1 are applied.

The zeros of the system establish the form of $u_{d}$, which, in this case, it is determined by $k_{d}=1, k_{i}=0, p=0$ leading to:

$$
u_{d}(t)=-\alpha_{1} f-\alpha_{2} \dot{f}-\gamma_{1} f-\gamma_{2} \dot{f}
$$

with $\alpha_{1}=-1, \alpha_{2}=-5$ given by $(21)$ and $\gamma_{1}=3.375$, $\gamma_{2}=6.75$ given by $(23)$. The final term $u_{d}$ results in:

$$
u_{d}=-1.75 \hat{f}-2.375 \hat{\dot{f}} .
$$

where the estimations are obtained by (7) with poles placed at $s=-10$. 

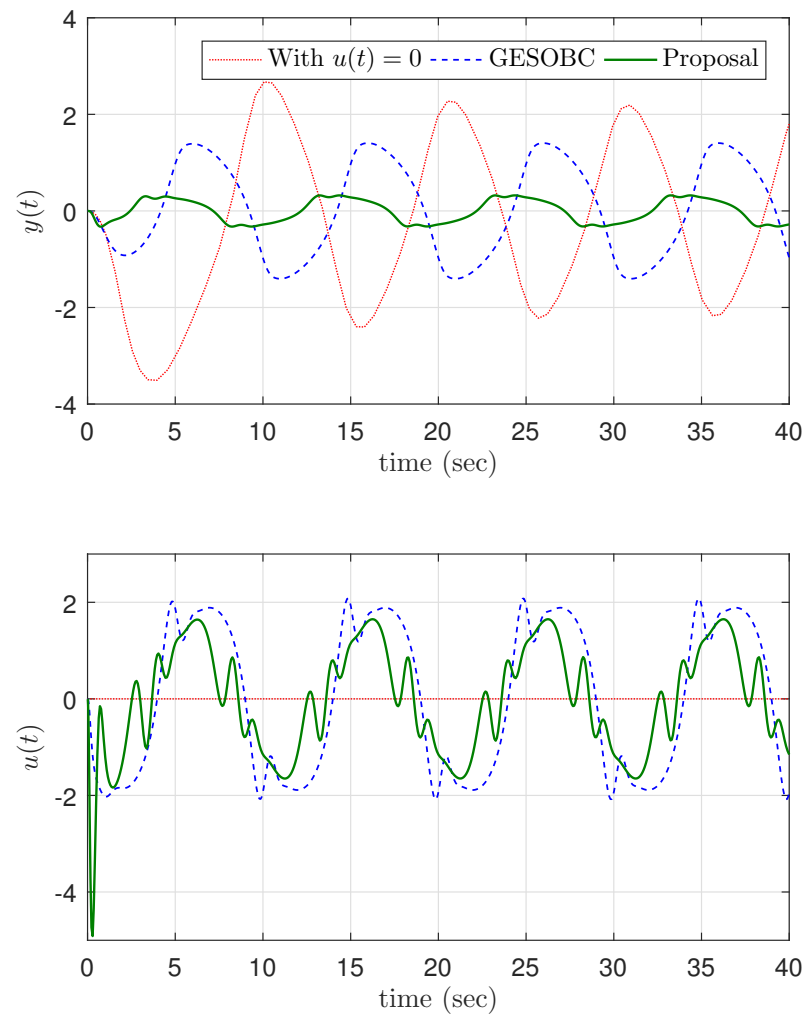

Figure 4: Simulation of Example 3.

It is not hard to see that the GESO-based control law, which is constructed by imposing the steady-state in (36), leads to:

$$
u_{d}=-1.75 \hat{f} \text {. }
$$

A simulation result is presented in Figure 4. It can be seen how, with the proposed method, the disturbance rejection capabilities of $u_{d}(t)$ are enhanced. This is because $\dot{f}$ has a strong effect in (37). See how the generated control action (green line) has small oscillations when compared with the GESOBC (dashed blue line). This is precisely the effect of $\hat{\dot{f}}$ in $(37)$.

As a matter of fact, if the conventional ESO is applied to this system in the same way as in (33), the closed loop is found to be unstable.

\section{Conclusions and Future Works}

This paper presents an ESO-based control strategy to solve the disturbance attenuation problem for a class of non integral-chain systems subject to non-linear mismatched uncertainties. In order to improve these results, future works could be focused in providing a closed-loop stability analysis relaxing the assumptions which have been imposed. Another research direction is the design of new observers being able to accurately estimate all the states and disturbance derivatives, even in the presence of high-frequency disturbances. Finally, the proposal could be extended to deal with MIMO systems with multiple uncertainties, to handle uncertainties in the system nominal matrices.

\section{Appendix A. Proof of Proposition 1}

Let us set

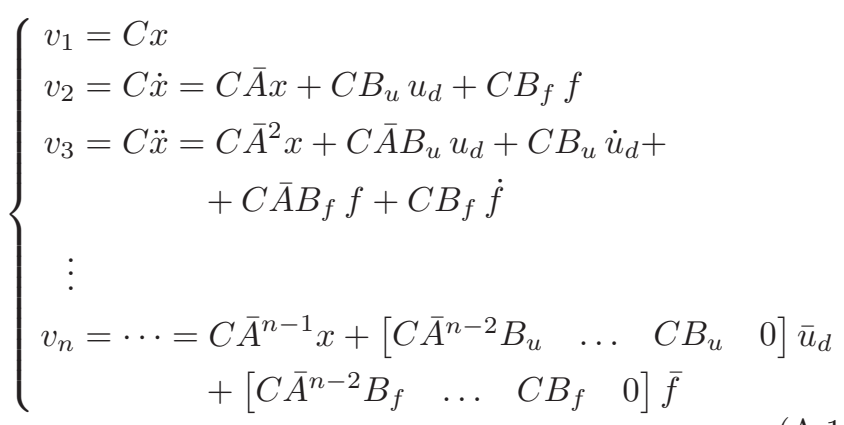

being $v_{i}=y^{(i-1)}$. System (A.1) can be written in matrix form:

$$
v=\mathcal{O} x+\mathcal{U} \bar{u}_{d}+\mathcal{F} \bar{f}
$$

where, by Assumption 2, $x$ can be obtained as

$$
x=\mathcal{O}^{-1} v-\mathcal{O}^{-1} \mathcal{U} \bar{u}_{d}-\mathcal{O}^{-1} \mathcal{F} \bar{f} .
$$

Obviously, (A.1) has been constructed to satisfy

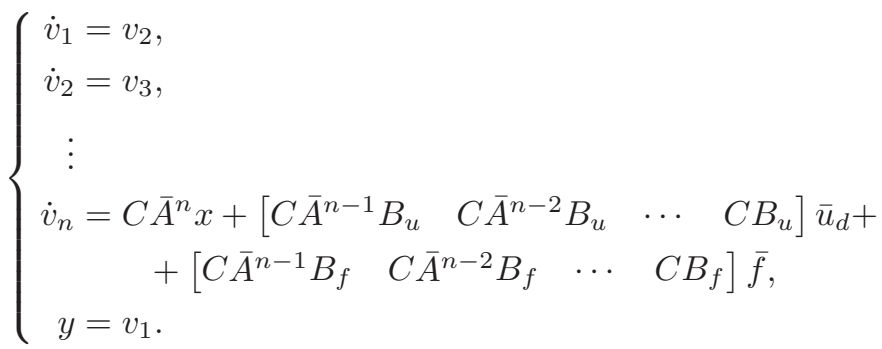

where, by substituting (A.3), leads to (10)-(11).

\section{Appendix B. Proof of Lemma 1}

1). It is well-known [29] that

$$
\begin{aligned}
& C \bar{A}^{k} B_{u}=0, \forall k<n-m_{u}^{t o t}-1 \\
& C \bar{A}^{k} B_{f}=0, \forall k<n-m_{f}^{t o t}-1 .
\end{aligned}
$$

Therefore, the prove immediately follows as it is straightforward to show that

$$
\begin{aligned}
C \bar{A}^{n} \mathcal{O}^{-1} \mathcal{U} & =\left[\begin{array}{llllll}
\bar{b}_{u, 0} & \ldots & \bar{b}_{u, m_{u}^{\text {tot }}} & 0 & \ldots & 0
\end{array}\right], \\
C \bar{A}^{n} \mathcal{O}^{-1} \mathcal{F} & =\left[\begin{array}{llllll}
\bar{b}_{f, 0} & \ldots & \bar{b}_{f, m_{f}^{t o t}} & 0 & \ldots & 0
\end{array}\right] .
\end{aligned}
$$

2). System (9) can be seen as one of the multiples statespace realizations of the following differential equation

$$
\begin{aligned}
y^{(n)}+a_{1} y^{(n-1)}+\ldots+a_{n} y= & \beta_{0} u+\ldots+\beta_{n-1} u^{(n-1)}+ \\
& +\gamma_{0} f+\ldots+\gamma_{n-1} f^{(n-1)} .
\end{aligned}
$$


The zeros of $\left(\bar{A}, B_{u}, C\right)$ and $\left(\bar{A}, B_{f}, C\right)$ are determined by the roots of $\beta_{0}+\beta_{1} s+\ldots+\beta_{n-1} s^{n-1}$ and $\gamma_{0}+\gamma_{1} s+$ $\ldots+\gamma_{n-1} s^{n-1}$, respectively. By writing (B.1) as

$$
\begin{aligned}
y^{(n)}= & -a_{1} y^{(n-1)}-\ldots-a_{n} y+\beta_{0} u+\ldots+\beta_{n-1} u^{(n-1)} \\
& +\gamma_{0} f+\ldots+\gamma_{n-1} f^{(n-1)}
\end{aligned}
$$

it can be seen that it exactly match with the last state equation in (10) with the coefficients $\beta_{i}, \gamma_{i}$, identified with $b_{u, i}, b_{f, i}$ in (11), respectively.

\section{References}

[1] W. H. Chen, J. Yang, L. Guo, S. Li, Disturbance-observerbased control and related methods-An overview, IEEE Transactions on Industrial Electronics 63 (2) (2016) 1083-1095.

[2] Z. Gao, On the centrality of disturbance rejection in automatic control, ISA transactions 53 (4) (2014) 850-857.

[3] B. Z. Guo, Z.-H. Wu, Output tracking for a class of nonlinear systems with mismatched uncertainties by active disturbance rejection control, Systems \& Control Letters 100 (2017) 21-31.

[4] K. Ohishi, M. Nakao, K. Ohnishi, K. Miyachi, Microprocessorcontrolled dc motor for load-insensitive position servo system, IEEE Transactions on Industrial Electronics IE-34 (1) (1987) $44-49$.

[5] W. H. Chen, D. J. Ballance, P. J. Gawthrop, J. O'Reilly, A nonlinear disturbance observer for robotic manipulators, IEEE Transactions on industrial Electronics 47 (4) (2000) 932-938.

[6] J. H. She, M. Fang, Y. Ohyama, H. Hashimoto, M. Wu, Improving disturbance-rejection performance based on an equivalentinput-disturbance approach, IEEE Transactions on Industrial Electronics 55 (1) (2008) 380-389.

[7] J. H. She, X. Xin, Y. Pan, Equivalent-input-disturbance approach-Analysis and application to disturbance rejection in dual-stage feed drive control system, IEEE/ASME Transactions on Mechatronics 16 (2) (2011) 330-340.

[8] Q.-C. Zhong, D. Rees, Control of uncertain lti systems based on an uncertainty and disturbance estimator, Journal of Dynamic Systems, Measurement, and Control(Transactions of the ASME) 126 (4) (2004) 905-910.

[9] Q. C. Zhong, A. Kuperman, R. Stobart, Design of ude-based controllers from their two-degree-of-freedom nature, International Journal of Robust and Nonlinear Control 21 (17) (2011) 1994-2008.

[10] A. Castillo, R. Sanz, P. Garcia, P. Albertos, Robust design of the uncertainty and disturbance estimator, IFAC-PapersOnLine 50 (1) (2017) 8262-8267.

[11] Z. Gao, Y. Huang, J. Han, An alternative paradigm for control system design, in: Decision and Control, 2001. Proceedings of the 40th IEEE Conference on, Vol. 5, IEEE, 2001, pp. 45784585.

[12] H. Jingqing, The extended state observer of a class of uncertain systems, Control and Decision 1 (1995) 19-23 (In Chinese).

[13] S. Li, J. Yang, W. H. Chen, X. Chen, Generalized extended state observer based control for systems with mismatched uncertainties, IEEE Transactions on Industrial Electronics 59 (12) (2012) 4792-4802.

[14] C. Zhang, S. He, Generalized output feedback active disturbance rejection control for uncertain lower-triangular nonlinear systems, in: Control Conference (CCC), 2016 35th Chinese, IEEE, 2016, pp. 533-538.

[15] J. Yang, A. Zolotas, W.-H. Chen, K. Michail, S. Li, Robust control of nonlinear maglev suspension system with mismatched uncertainties via dobc approach, ISA transactions 50 (3) (2011) 389-396.

[16] W.-H. Chen, Nonlinear disturbance observer-enhanced dynamic inversion control of missiles, Journal of Guidance, Control, and Dynamics 26 (1) (2003) 161-166.
[17] Y. A.-R. I. Mohamed, Design and implementation of a robust current-control scheme for a pmsm vector drive with a simple adaptive disturbance observer, IEEE transactions on industrial electronics 54 (4) (2007) 1981-1988.

[18] J. Han, From pid to active disturbance rejection control, IEEE transactions on Industrial Electronics 56 (3) (2009) 900-906.

[19] Z. Gao, Active disturbance rejection control: a paradigm shift in feedback control system design, in: American Control Conference, 2006, IEEE, 2006, pp. 7-pp.

[20] B. Z. Guo, Z. L. Zhao, On the convergence of an extended state observer for nonlinear systems with uncertainty, Systems \& Control Letters 60 (6) (2011) 420-430.

[21] Z. Pu, R. Yuan, J. Yi, X. Tan, A class of adaptive extended state observers for nonlinear disturbed systems, IEEE Transactions on Industrial Electronics 62 (9) (2015) 5858-5869.

[22] W. Xue, Y. Huang, On performance analysis of ADRC for a class of MIMO lower-triangular nonlinear uncertain systems, ISA transactions 53 (4) (2014) 955-962.

[23] Q. Zheng, L. Q. Gaol, Z. Gao, On stability analysis of active disturbance rejection control for nonlinear time-varying plants with unknown dynamics, in: Decision and Control, 2007 46th IEEE Conference on, IEEE, 2007, pp. 3501-3506.

[24] A. Castillo, R. Sanz, P. Garcia, P. Albertos, A quaternion-based and active disturbance rejection attitude control for quadrotor, in: Information and Automation (ICIA), 2016 IEEE International Conference on, IEEE, 2016, pp. 240-245.

[25] S. E. Talole, J. P. Kolhe, S. B. Phadke, Extended-stateobserver-based control of flexible-joint system with experimental validation, IEEE Transactions on Industrial Electronics 57 (4) (2010) 1411-1419.

[26] Y. X. Su, B. Y. Duan, C. H. Zheng, Y. Zhang, G. Chen, J. Mi, Disturbance-rejection high-precision motion control of a stewart platform, IEEE transactions on control systems technology 12 (3) (2004) 364-374.

[27] D. Wu, K. Chen, Design and analysis of precision active disturbance rejection control for noncircular turning process, IEEE Transactions on Industrial Electronics 56 (7) (2009) 2746-2753.

[28] R. Zhou, W. Tan, A generalized active disturbance rejection control approach for linear systems, in: Industrial Electronics and Applications (ICIEA), 2015 IEEE 10th Conference on, IEEE, 2015, pp. 248-255.

[29] A. Isidori, Nonlinear control systems, Springer Science \& Business Media, 2013.

[30] S. Chen, W. Bai, Y. Huang, Adrc for systems with unobservable and unmatched uncertainty, in: Control Conference (CCC), 2016 35th Chinese, IEEE, 2016, pp. 337-342.

[31] S. N. Pawar, R. Chile, B. Patre, Design of generalized extended state observer based control for nonlinear systems with matched and mismatched uncertainties, in: Control Conference (ICC), 2017 Indian, IEEE, 2017, pp. 65-71.

[32] Y. Huang, W. Xue, Active disturbance rejection control: methodology and theoretical analysis, ISA transactions 53 (4) (2014) 963-976. 